\title{
Foreign Body Embedded in the Schneiderian Membrane
}

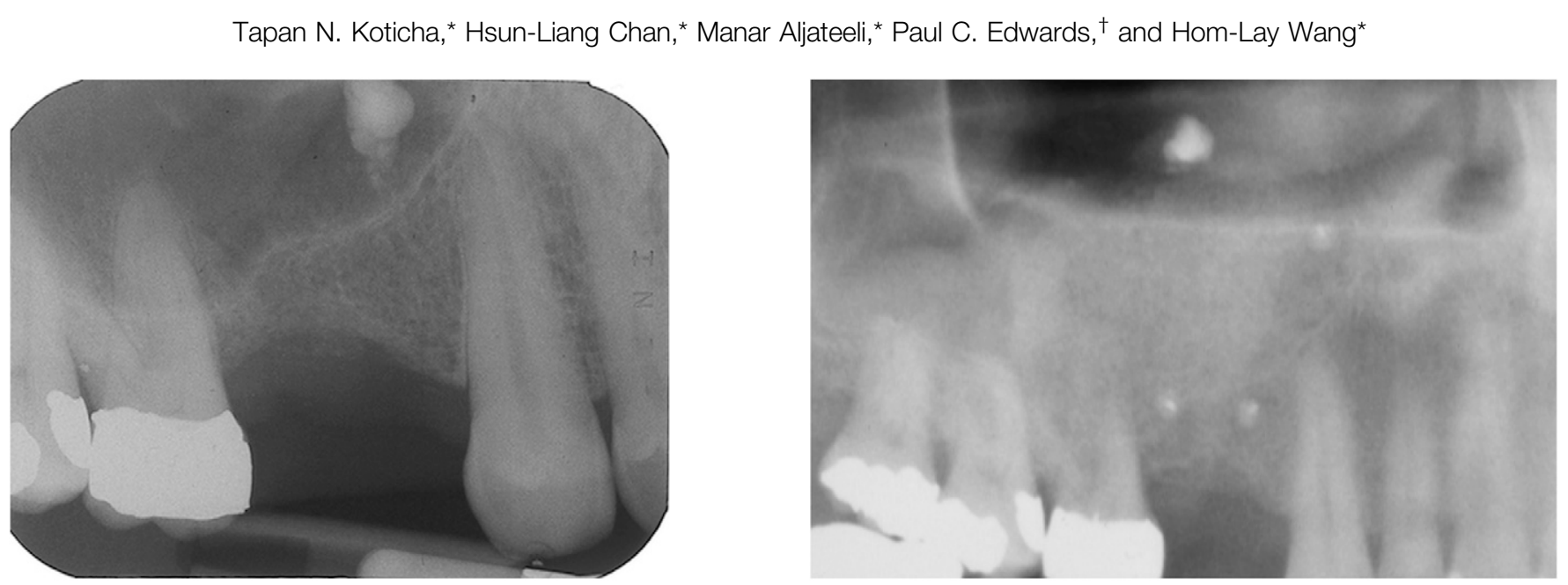

Introduction: Foreign bodies have been reported in the maxillary sinus. It has been suggested that asymptomatic foreign bodies do not require removal. The effect of such retained foreign bodies on sinus-elevation procedures is unknown.

Case Presentation: This case report documents the management of an asymptomatic foreign body embedded in the Schneiderian membrane. The foreign body was left in situ while elevating the membrane for a sinus-augmentation procedure. A staged approach with delayed implant placement was used. Leaving the foreign body in situ did not seem to adversely affect the healing or clinical success of the augmentation procedure.

Conclusions: From this short-term follow-up case, a retained foreign body in the maxillary sinus consistent with periapical endodontic filling material could be left in place in conjunction with maxillary sinus grafting based on a lack of symptoms. Long-term clinical success has yet to be confirmed. Clin Adv Periodontics 2014;4:135-138.

Key Words: Foreign bodies; maxillary sinus; nasal mucosa; sinus floor augmentation.

\section{Background}

The presence of foreign bodies in the maxillary sinus is relatively rare. ${ }^{1}$ Many foreign bodies in the sinus are of iatrogenic origin (e.g., displacement of teeth or restorative materials into the sinus $)^{2-4}$ or attributable to leakage of impression material or other objects through a patent oroantral fistula ${ }^{5}$ or traumatic injuries. ${ }^{6}$ The presence of these foreign bodies may result in chronic sinusitis and/or secondary infection and rarely may present with an ominous appearance. ${ }^{7}$ However, asymptomatic foreign bodies have been reported, and the necessity for routine surgical removal has been questioned. ${ }^{8}$ The decision

\footnotetext{
* Department of Periodontics and Oral Medicine, School of Dentistry, University of Michigan, Ann Arbor, MI.

+ Currently, Department of Oral Pathology, Medicine and Radiology, School of Dentistry, Indiana University, Indianapolis, IN; previously, Department of Periodontics and Oral Medicine, School of Dentistry, University of Michigan.
}

Submitted September 23, 2012; accepted for publication November 14, 2012

doi: 10.1902/cap.2012.120104 whether to remove asymptomatic foreign bodies might depend on biocompatibility (e.g., reactive or inert) and history (e.g., how long the material has been in place) of foreign materials. In this case report, the management of an asymptomatic foreign body during sinus augmentation and dental implant placement is discussed.

\section{Clinical Presentation}

A 45-year-old male was referred to the Graduate Periodontics Clinic at the University of Michigan (Ann Arbor, Michigan) in May 2010 for sinus augmentation and dental implant placement at teeth \#4 and \#5. The patient was otherwise healthy and provided written consent to be treated. A radiograph revealed the presence of a radiopaque body at the sinus floor, in the region of tooth \#5, which had a history of an apicoectomy (Fig. 1). Tooth \#5 was extracted $\approx 7$ years ago because it was deemed non-restorable. The radiopaque mass was irregular in shape, with the appearance of extruded endodontic sealer. At the time of current presentation, the patient was clinically asymptomatic and free of any signs and symptoms of chronic sinusitis or other sinus-related problems. 

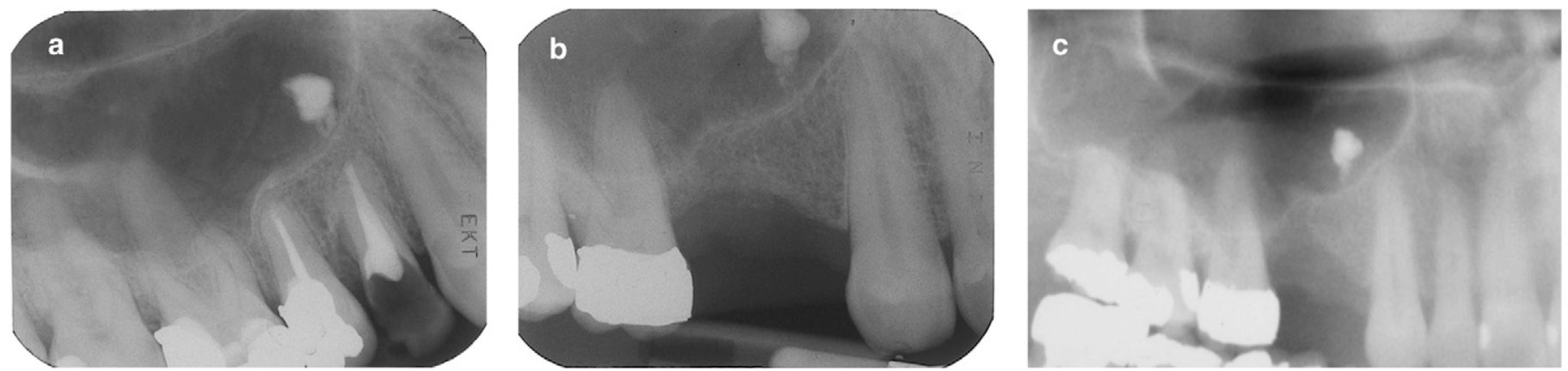

FIGURE 1a Periapical radiograph of the foreign body from 10 years ago. 1b and 1c Preoperative radiographs of the foreign body.
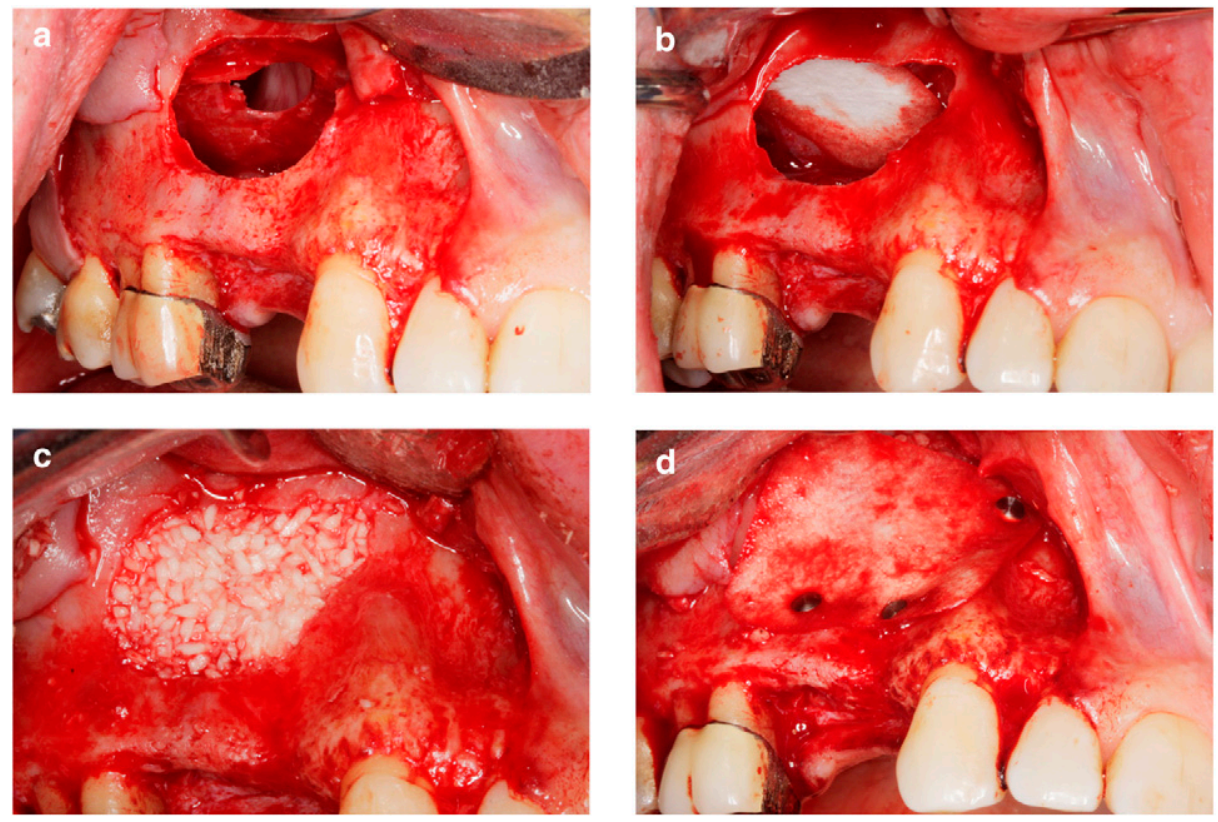

FIGURE 2a Accidental perforation of the Schneiderian membrane. $2 \mathrm{~b}$ Repair with a collagen membrane. 2c Placement of bone graft. 2d Coverage of lateral window with collagen membrane.

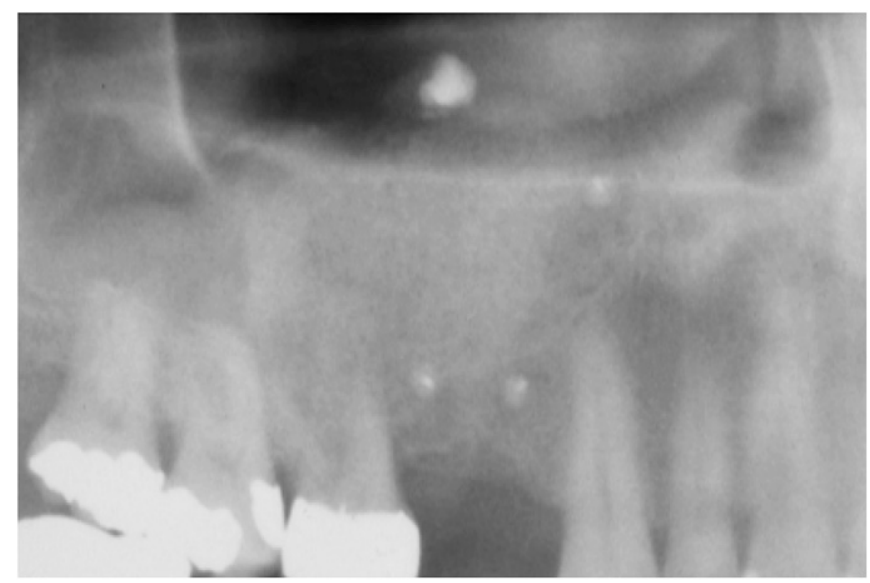

FIGURE 3 Radiograph after sinus augmentation. Note the elevated position of the foreign body, still embedded in the Schneiderian membrane.

\section{Case Management}

The clinical impression of the radiopaque mass was that of an iatrogenically introduced foreign body. The patient was referred to an otolaryngologist (Dr. Paul Tichenor, Internal Medicine, University of Michigan, Ann Arbor,
Michigan) to secure additional information and obtain clearance for a sinus-augmentation procedure. The otolaryngologist performed a computed tomography scan of the right maxillary sinus which revealed the absence of any aggressive features. The recommendation was that no additional treatment or evaluations were needed for the sinus. Clearance was given for a sinus-augmentation procedure. It was decided to explore the area for the foreign body after a lateral window access to the sinus. The membrane sustained a perforation during its elevation (Fig. 2a). After elevation, a thorough exploration revealed that the foreign body was embedded in the membrane. A decision was made to proceed without retrieval of the body because of its asymptomatic nature and the amount of breakdown of hard and soft tissues that would be needed to remove it. The perforation was repaired using a collagen membrane, ${ }^{\ddagger}$ and the sinus was augmented with an allograft $\$$ (Figs. 2b through $2 \mathrm{~d}$ and 3).

After a healing period of 6 months, two implants" were placed at teeth \#4 and \#5 (Fig. 4).

‡ BioMend, Zimmer Dental, Carlsbad, CA.

$\S$ Puros Cancellous Particulate Allograft, Zimmer Dental.

" NobelReplace Straight Groovy, Nobel Biocare, Yorba Linda, CA. 


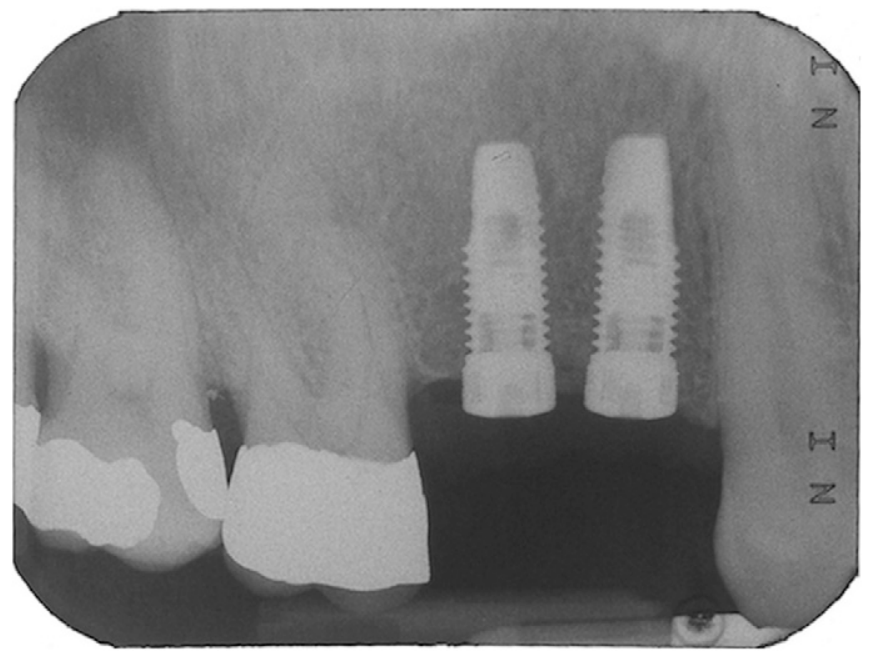

FIGURE 4 Radiograph of implants placed at teeth \#4 and \#5.

\section{Clinical Outcomes}

Healing was uneventful after both surgical procedures. No manifestations or symptoms of sinus pathology were detected throughout the follow-up period. The post-surgical radiograph shows the foreign body still embedded in the elevated membrane, at a higher level in the sinus (Fig. 3).
The implants were placed as dictated by restorative guidelines.

\section{Discussion}

This case report suggests that a foreign body in the maxillary sinus, if asymptomatic, does not necessarily require removal, especially if it has been present for an extended period of time (7 years in this case). The fact that the sinus was asymptomatic, despite the presence of the foreign body, was crucial to the decision to not remove it. If the foreign body had caused an immune reaction or an infection, the priority would have been to remove it. However, removal of such a body would result in extensive breakdown of the membrane. Although this breakdown is completely justified if the sinus was symptomatic (chronic sinusitis, radiographic evidence of membrane thickening, etc.), an asymptomatic membrane justifies the consideration of a conservative approach. ${ }^{8}$ The foreign body embedded in the Schneiderian membrane was undisturbed and a sinusaugmentation procedure was performed with eventual placement of implants. An attempt at removal of the foreign body would have resulted in breakdown of the membrane, thus delaying the subsequent procedures. Although this approach was successful in the short term, studies with larger numbers of cases are needed to recommend it routinely.

\section{Summary}

Why is this case new information?

- This case documents the successful augmentation of a maxillary sinus in the presence of an asymptomatic foreign body embedded in the Schneiderian membrane.

What are the keys to successful management of this case?
- The key to successful management is case selection. Only in cases in which the foreign body is localized and the sinus asymptomatic can the sinus-augmentation procedure be performed without overt risk of failure.

What are the primary limitations to success in this case?
- The primary limitation to success would have been the presence of an infection or an immune reaction in the sinus membrane.

\section{Acknowledgment}

The authors report no conflicts of interest related to this case report.
CORRESPONDENCE:

Dr. Hom-Lay Wang, 1011 North University Ave., Ann Arbor, MI 481091078. E-mail: homlay@umich.edu. 


\section{References}

1. Lana JP, Carneiro PM, Machado Vde C, de Souza PE, Manzi FR, Horta MC. Anatomic variations and lesions of the maxillary sinus detected in cone beam computed tomography for dental implants. Clin Oral Implants Res 2012;23:1398-1403.

2. Mohanavalli S, David JJ, Gnanam A. Rare foreign bodies in oro-facial regions. Indian J Dent Res 2011;22:713-715.

3. Smith JL 2nd, Emko P. Management of a maxillary sinus foreign body (dental bur). Ear Nose Throat J 2007;86:677-678.

4. Costa A, Branca V, Guzzi G. Dental amalgam in the maxillary sinus. $\mathrm{Br}$ J Radiol 2008;81:987.

indicates key reference.
5. Rodrigues MT, Munhoz ED, Cardoso CL, de Freitas CA, Damante JH. Chronic maxillary sinusitis associated with dental impression material. Med Oral Patol Oral Cir Bucal 2009;14:E163E166.

6. Gupta AC, Murthy DP, Pulotu ML. Unusual type of foreign body in the maxillary sinus. J Laryngol Otol 1990;104:718-719.

7. Macan D, Cabov T, Kobler P, Bumber Z. Inflammatory reaction to foreign body (amalgam) in the maxillary sinus misdiagnosed as an ethmoid tumor. Dentomaxillofac Radiol 2006;35:303-306.

8. Selvi F, Enoz M, Yazgin I, Cakarer S, Keskin C. Do asymptomatic foreign bodies in the maxillary sinus always need to be removed? $B$ ENT 2008;4:243-247. 